\title{
Регулярные и вполне регулярные дифференциальные операторы
}

\author{
Е. А. Ширяев, А. А. Шкаликов
}

1. Регулярные операторы. В работе [4 Биркгоф выделил класс обыкновенных дифференциальных операторов, для которых получил оценки ядер Грина и доказал теорему о разложении по собственным функциям. Операторы из этого класса он назвал регулярными. Напомним определение Биркгофа в упрощенной форме.

Пусть $L-$ оператор, порожденный дифференциальным выражением

$$
l(y)=(-i)^{n} y^{(n)}(x)+p_{2}(x) y^{(n-2)}+\cdots+p_{n}(x) y,
$$

и $n$ линейно независимыми краевыми условиями вида

$$
U_{j}(y)=\sum_{s=0}^{n-1}\left(a_{j, s} y^{(s)}(0)+b_{j, s} y^{(s)}(1)\right)=0, \quad j=1, \ldots, n .
$$

Предполагаем, что коэффициенты $p_{j}(x), j=1, \ldots, n,-$ суммируемые комплексные функции на отрезке $[0,1]$. Считаем, что $L$ действует в пространстве $L_{2}(0,1)$ и определен равенством $L(y)=l(y)$ на области

$D(L)=\left\{y \mid y^{(s)} \in A C[0,1], s=0,1, \ldots, n-1, l(y) \in L_{2}, U_{j}(y)=0, j=1, \ldots, n\right\}$.

Здесь $A C[0,1]$ - пространство абсолютно непрерывных функций.

Перепишем краевые условия, выделив старшие производные

$$
U_{j}(y):=a_{j} y^{\left(k_{j}\right)}(0)+b_{j} y^{\left(k_{j}\right)}(1)+\sum_{s=0}^{k_{j}-1}\left(a_{j, s} y^{(s)}(0)+b_{j, s} y^{(s)}(1)\right)=0, \quad j=1, \ldots, n .
$$

Число $k_{j}\left(j=1, \ldots, n, 0 \leqslant k_{j} \leqslant n-1\right)$ назовем порядком краевого условия, а число $\varkappa=k_{1}+\ldots+k_{n}-$ суммарным порядком. Заменим краевые условия их линейными комбинациями, при которых суммарный порядок наименьший. Считаем, что получившиеся краевые условия имеют вид (3), причем нумерация такова, что $n-1 \geqslant k_{1} \geqslant k_{2} \geqslant \ldots \geqslant k_{n} \geqslant 0$. Тогда старшие линейные формы

$$
U_{j}^{0}(y)=a_{j} y^{\left(k_{j}\right)}(0)+b_{j} y^{\left(k_{j}\right)}(1), \quad j=1, \ldots, n,
$$

также линейно независимы (иначе суммарный порядок можно понизить), что влечет $k_{j}>k_{j+2}, a_{j} b_{j} \neq 0$.

Работа выполнена при поддержке Российского фонда фундаментальных исследований, грант № 04-01-00712 и программы «Ведущие научные школы», грант № НШ-5247.2006.1. 
Определение. При четном $n=2$ оператор $L$ назовем регулярным по Биркгобу, если не равен нулю определитель

$$
\theta=\left|\begin{array}{ccccccc}
a_{1} \varepsilon_{1}^{k_{1}} & \ldots & a_{1} \varepsilon_{m-1}^{k_{1}} & a_{1} \varepsilon_{m}^{k_{1}} & b_{1} \varepsilon_{m+1}^{k_{1}} & \ldots & b_{1} \varepsilon_{n}^{k_{1}} \\
\cdot & \cdot & \cdot & \cdot & \cdot & \cdot & \cdot \\
a_{n} \varepsilon_{1}^{k_{n}} & \ldots & a_{n} \varepsilon_{m-1}^{k_{n}} & a_{n} \varepsilon_{m}^{k_{n}} & b_{n} \varepsilon_{m+1}^{k_{n}} & \ldots & b_{n} \varepsilon_{n}^{k_{n}}
\end{array}\right|
$$

где $\varepsilon_{k}=\exp (2 \pi(k-1) i / n), k=1, \ldots, n,-$ корни $n$-й степени из 1 . При нечетном $n=2 m-1$ требуем, чтобы помимо этого определителя был также отличен от нуля определитель, получающийся заменой в $a_{j}$ на $b_{j}$

При $n=2 m$ приведенное определение отличается от классического [4], [9], где требуется отличие от нуля еще одного числа, но несложно показать, что второе число может обращаться в нуль только одновременно с приведенным здесь первым числом. Коэффициенты $p_{j}(x)$ дифференциального выражения и младшие члены в краевых условиях не участвуют в определении регулярности; $L$ регулярен тогда и только тогда, когда регулярен оператор $L_{0}(y)=(-i)^{n} y^{(n)}$, порожденный краевыми условиями $U_{j}^{0}(y)=0, j=1, \ldots, n$.

Определение регулярности переносится на существенно более общие классы краевых задач со спектральным параметром. Для обыкновенных дифференциальных уравнений и систем это сделано в [14], [12], [7], а теория регулярных задач для уравнений с частными производными построена в работах [6], 1, [2. Интересно отметить, что для обыкновенных дифференциальных операторов порядка $2 m$ в случае распадающихся краевых условий можно определить регулярность по Лопатинскому и оно будет эквивалентным регулярности по Биркгофу (см. [13, § 9]).

Для формулировки основной теоремы о регулярных операторах удобно ввести дополнительные обозначения. Лучи $\arg \rho= \pm\left(\pi / 2 n+\arg \left(i \varepsilon_{k}\right)\right)$ в комплексной $\rho$-плоскости назовем критическими. Их число равно $n$ и $2 n$ в четном и нечетном случаях, соответственно. Вырежем из $\rho$-плоскости открытые секторы раствора $\varepsilon<\pi /(2 n)$, биссектрисами которых служат критические лучи, а оставшиеся $n$ или $2 n$ замкнутых секторов обозначим через $\Omega(\varepsilon)$.

Если резольвентное множество оператора $L$ непусто, то из компактности вложения $D(L) \subset L_{2}(0,1)$ (мы снабжаем $D(L)$ нормой графика оператора $L$, тогда $D(L)$ становится гильбертовым пространством) следует, что спектр $L$ дискретный, т.е. состоит из изолированных собственных значений $\left\{\lambda_{k}\right\}_{1}^{\infty}$ конечной алгебраической кратности. Обозначим через $\rho_{k, j}, k=1, \ldots, n$, корни уравнения $\lambda_{j}=\rho^{n}$, через $B_{k, j}(\delta)$ - круги радиуса $\delta$ в $\rho$-плоскости с центрами в точках $\rho_{k, j}$, и через $B(\delta)$ - объединение всех таких кругов по обоим индексам $k$ и $j$. Известно [9], что резольвента $L$ есть интегральный оператор

$$
\left(L-\rho^{n}\right)^{-1} f(x)=\int_{0}^{1} G(x, \xi, \rho) f(\xi) d \xi,
$$

где $G(x, \xi, \rho)$ - ядро Грина. Теперь сформулируем основной результат.

Теорема 1. Следующие утверждения эквивалентны. 
1) Оператор L регулярен по Биркгофу;

2) при любом $\delta>0$ всякий некритический луч, выходящий из нуля, асимптотически не пересекает множество $\mathbb{C} \backslash B(\delta)$, и для всех $x, \xi \in[0,1]$ и $\rho \in \mathbb{C} \backslash B(\delta)$ для функиии Грина справедлива оценка

$$
|G(x, \xi, \rho)| \leqslant M|\rho|^{-n+1}
$$

где постоянная $M=M(\delta)$ не зависит от $x, \xi, \rho$;

3) найдется последовательность точек $\left\{\rho_{k}\right\}$ в одном из секторов множества $\Omega(\varepsilon)$ (в случае нечетного $n-$ две последовательности в двух соседних секторах этого множества), такая, что $\rho_{k} \rightarrow \infty$ и оценка (4) выполнена при $\rho=\rho_{k}$;

4) при любом $\varepsilon>0$ в области $\Omega(\varepsilon)$ выполняется оценка

$$
\left\|\left(L-\rho^{n}\right)^{-1}\right\| \leqslant M|\rho|^{-n}, \quad|\rho| \geqslant\left|\rho_{0}\right|,
$$

где постоянная $M=M\left(\varepsilon, \rho_{0}\right)$ не зависит от $\rho$, a $\|\cdot\|$ означает норму в $L_{2}$;

5) найдется последовательность точек $\left\{\rho_{k}\right\}$ в одном из секторов множества $\Omega(\varepsilon)$ (в случае нечетного $n-$ две последовательности в двух соседних секторах этого множества), такая, что $\rho_{k} \rightarrow \infty$ и оценка (5) выполнена при $\rho=\rho_{k}$;

6) система собственных и присоединенных функиий оператора L образует безусловный базис со скобками в пространстве $L_{2}$, причем в скобки объединяются не более двух собственных функиий.

ДокаЗАТЕЛьСтво. Импликация 1) $\Rightarrow$ 2) доказана Биркгофом [4. Формально в [4] (см. также [9]) оценка (4) доказана на некоторой последовательности контуров, уходящих на бесконечность. Однако при условии регулярности, оценку снизу характеристического определителя (голоморфной функции, нули которой совпадают с числами $\rho_{k, j}$ ) легко провести вне множества $B(\rho)$. Тогда оценка (44) получается при всех $\rho \in \mathbb{C} \backslash B(\rho)$. Импликация 1$) \Rightarrow 4)$, доказана Бензингером [3]. Импликации 2) $\Rightarrow 3$ ) и 4) $\Rightarrow$ 5) тривиальны, а 5) $\Rightarrow$ 1) доказана Минкиным в его недавней работе [8. Импликация 1) $\Rightarrow 6$ ) доказана Шкаликовым [1] (там же см. ссылки на предшествующие работы Н. Данфорда и Дж. Шварца, Г. М. Кесельмана и В. П. Михайлова на эту тему). Остается доказать 4$) \Rightarrow 1$ ) и 6 ) $\Rightarrow 1$ ). Доказательства обеих этих импликаций нетривиальны, в них существенно используются результаты и приемы недавней работы Минкина 8]. Подробное изложение будет дано в другой работе, здесь отметим только один вспомогательный результат, играющий важную роль.

Лемма. Пусть $\left\{\alpha_{k}\right\}_{1}^{\infty}-$ комплексные, не равные нулю числа, аргументы которых различны. Положим

$$
\psi_{j}(x)=\sum_{k=1}^{n} c_{k, j} e^{\alpha_{k} \rho_{j} x}\left(1+\varphi_{k, j}(x)\right)
$$


где $c_{k, j}$ - произвольнье числа, такие что $\left|c_{1, j}\right|+\ldots+\left|c_{n, j}\right| \neq 0, a\left\|\varphi_{k, j}\right\|^{2}=$ $o\left(\left|\rho_{j}\right|^{-1}\right)$ при $\left|\rho_{j}\right| \rightarrow \infty$. Назовем критическими лучи, аргументы которых равнь $\pm \pi / 2+\arg \alpha_{k}$. Последовательность $\left\{\rho_{j}\right\}_{1}^{\infty}$, занумерованную в порядке возрастания модулей, назовем редкой, если найдется челое число l, такое, что $\left|\rho_{j+l} / \rho_{j}\right| \geqslant 2$ при всех $j \geqslant 1$.

Пусть система $\left\{\psi_{j}(x)\right\}_{1}^{\infty}$ образует базис Рисса со скобками в замыкании своей линейной оболочки в пространстве $L_{2}(0,1)$, причем в скобки заключаются не более фиксированного числа $N$ функиий. Тогда в каждом замкнутом секторе, не содержащем критические лучи, последовательность $\left\{\rho_{j}\right\}$ является редкой.

2. Вполне регулярные операторы. Здесь будем рассматривать дифференциальные операторы четного порядка $n=2 m$, заданные дифференциальным выражением

$$
l(y)=\sum_{k=0}^{m}(-1)^{k}\left\{\left(p_{k}(x) y^{(k)}\right)^{(k)}-\left[\left(q_{k}(x) y^{(k)}\right)^{(k-1)}+\left(r_{k}(x) y^{(k-1)}\right)^{(k)}\right]\right\}
$$

где $p_{m}(x)=1, r_{0}(x)=0$. Чтобы не осложнять существо дела, предположим, что коэффициенты $p_{k}(x), q_{k}(x), r_{k}(x)$ таковы, что после раскрытия производных дифференциальное выражение приводится к виду (11). Для этого достаточно, чтобы $p_{k}(x), r_{k}(x) \in W_{1}^{k}[0,1], q_{k}(x) \in W_{1}^{k-1}[0,1]$.

Введем квазипроизводные

$$
\begin{array}{r}
y^{[k]}=y^{(k)}, \quad k=0, \ldots, m-1, \quad y^{[m]}=y^{(m)}-r_{m} y^{(m-1)}, \\
y^{[m+k]}=-\left(y^{[m+k-1]}\right)^{\prime}+p_{m-k} y^{(m-k)}+\left[q_{m-k+1} y^{(m-k+1)}-r_{m-k} y^{(m-k-1)}\right], \\
k=1, \ldots, m .
\end{array}
$$

Легко видеть, что при фиксированном $x$ функционал $y^{[k]}(x)$ является линейной комбинацией функционалов $y^{(s)}(x), s=0, \ldots, k$, и наоборот. Поэтому краевые условия можно записать в виде

$$
B y^{\wedge}+C y^{\vee}=0
$$

где $B$ и $C$ - некоторые матрицы, а $y^{\wedge}$ и $y^{\vee}-$ векторы значений квазипроизводных:

$$
\begin{gathered}
y^{\wedge}=\left(y(0), y^{\prime}(0), \ldots, y^{(m-1)}(0), y(1), y^{\prime}(1), \ldots, y^{(m-1)}(1)\right)^{\mathrm{t}}, \\
y^{\vee}=\left(y^{[2 m-1]}(0), y^{[2 m-2]}(0), \ldots, y^{[m]}(0),-y^{[2 m-1]}(1),-y^{[2 m-2]}(1), \ldots,-y^{[m]}(1)\right)^{\mathrm{t}},
\end{gathered}
$$

где верхний индекс $\mathrm{t}$ означает транспонирование.

Определение. Оператор L, порожденный дифберенииалъным выражением (6) и краевыми условиями (8), назовем вполне регулярным, если выполнено условие $B^{-1}(\operatorname{im} C)=\mathbb{C}^{2 m} \ominus \operatorname{ker} C$, где $B^{-1}$ понимается как взятие полного прообраза от $\operatorname{im} C$ при отображсении $B$. 
Теорема 2. Пусть оператор L задан выражением (6) и краевыми условиями (8). Следующие утверждения эквивалентны .

1) Оператор L является вполне регулярным.

2) Числовой образ оператора $L$ не совпадает со всей комплексной плоскостью.

3) Числовой образ оператора $L$ содержится в некоторой полуплоскости в $\mathbb{C}$.

4) Квадратичная форма оператора $(L y, y)_{L_{2}}$ представима в виде

$$
\sum_{k=1}^{m}\left[\left(p_{k} y^{(k)}, y^{(k)}\right)_{L_{2}}+\left(q_{k} y^{(k)}, y^{(k-1)}\right)_{L_{2}}-\left(r_{k} y^{(k-1)}, y^{(k)}\right)_{L_{2}}\right]+\left(A y^{\wedge}, y^{\wedge}\right)_{\mathbb{C}^{2 m}}
$$

ДокАЗАТЕЛЬСтвО. Эквивалентность утверждений 2) и 3) следует из свойства выпуклости числового образа.

Импликация 1) $\Rightarrow 4$ ) для случая $q_{k}=r_{k} \equiv 0$ доказана в работе [15]. В общем случае доказательство можно провести аналогично. Чтобы показать справедливость обратного утверждения докажем следующую лемму.

Лемма. Пусть матрица $A$ такова, что для всех $x, y_{1}, y_{2} \in \mathbb{C}^{2 m}$ справедливо равенство $\left(y_{2}, x\right)_{\mathbb{C}^{2 m}}=\left(A y_{1}, x\right)_{\mathbb{C}^{2 m}}$, если $x \in B^{-1}(\operatorname{im} C)$ и $B y_{1}+C y_{2}=0$. Тогда оператор L вполне регулярен.

ДокаЗАтельство. Сначала покажем, что $B^{-1}(\operatorname{im} C) \perp \operatorname{ker} C$. Пусть векторы $y_{1}$ и $y_{2}$ таковы что $B y_{1}+C y_{2}=0$. Тогда $y_{1} \in B^{-1}(\operatorname{im} C)$ и $\left(y_{2}, y_{1}\right)_{\mathbb{C}^{2 m}}=$ $\left(A y_{1}, y_{1}\right)_{\mathbb{C}^{2 m}}$. Поскольку $B y_{1}+C\left(y_{2}+v\right)=0$ для любого $v \in \operatorname{ker} C$, то $\left(y_{2}+\right.$ $\left.v, y_{1}\right)_{\mathbb{C}^{2 m}}=\left(A y_{1}, y_{1}\right)_{\mathbb{C}^{2 m}}$. Поэтому $\left(v, y_{1}\right)_{\mathbb{C}^{2 m}}=0$, т.е. $B^{-1}(\operatorname{im} C) \perp \operatorname{ker} C$.

Равенство $B^{-1}(\operatorname{im} C)=\mathbb{C}^{2 m} \ominus \operatorname{ker} C$ следует теперь из того, что краевые условия линейно независимы.

Доказательство импликации 4) $\Rightarrow 3)$ следует из компактности вложений $W_{2}^{r} \subset W_{2}^{m}$ при $r \leqslant m-1$ и компактности операторов следа $T_{0} y=y^{(r)}(0)$ и $T_{1} y=y^{(r)}(1)$ как операторов из $W_{2}^{m}$ в $\mathbb{C}$. Доказательство обратной импликации более сложно и здесь мы его не приводим. В нем используется прием из работы [10].

Замечание. Всякий вполне регулярный оператор регулярен. Действительно, если L вполне регулярен, то его числовой образ лежит в некоторой полуплоскости, а потому найдется сектор в комплексной плоскости, в котором резольвента $\left(L-\rho^{m}\right)^{-1}$ имеет оценку (5). Тогда по теореме 1 оператор $L$ регулярен. Обратное, вообще говоря, неверно. Это показывает следующий пример.

Пример. Пусть $L y=y^{(4)}$, а краевые условия имеют вид

$$
\left\{\begin{aligned}
-y^{\prime \prime \prime}(0)+y^{\prime \prime}(0)+y(0) & =0 \\
y^{\prime \prime \prime}(1)+y(1) & =0 \\
y^{\prime}(0) & =0 \\
y^{\prime}(1) & =0
\end{aligned}\right. \text {. }
$$

Простая проверка показывает, что $L$ регулярен, но не вполне регулярен. 


\section{Список литературы}

[1] S. Agmon, S. Nirenberg. Properties of solutions of ordinary differential equations in Banach space// Commun. Pure Appl. Math., 15 (1962), p. 119-147.

[2] М. С. Агранович, М. И. Вишик. Эллиптические задачи с параметром и гиперболические задачи общего вида// УМН, 19, № 3, стр. 53-161.

[3] H. E. Benzinger. Green's function for ordinary differential operators// J. Diff. Equations, vol. 7, 1970, No. 3, p. 478-496.

[4] G. Birkhoff. Boundary Value and Expansion Problems of Ordinary Linear Differential Equations // T.A.S., 1908, oct, p. 373-395.

[5] Като. Теория возмущений линейных операторов//Москва, «Мир», 1972 г.

[6] Я. Б. Лопатинский. Об одном способе приведения граничных задач для систем дифберенциальных уравнений эллиптического типа и регулярные интегральные уравнения.// Украинский математический журнал, 5 (1953), стр. 123-151.

[7] Л. М. Лужина. Некоторые вопросы спектральной теории дифферециальных операторов на конечном интервале// Диссертация на соискание ученой степени кандидата физикоматематических наук. Москва, МГУ, 1991.

[8] A. M. Minkin. Resolvent's growth and Birkhoff-regularity// Journ. Math. Anal. Appl., v. 323 (2006), p. 387-402.

[9] М. А. Наймарк. Линейные дифференцальные операторы// Москва, «Наука», 1969.

[10] Е. А. Ширяев. Диссипативные краевые условия для обыкновенных дифференциальных операторов// Матем. заметки, 2005, том 77, выпуск 6, стр. 950-954.

[11] А. А. Шкаликов. Базисные свойства собственных функций обыкновенного дифференциального оператора// Успехи математических наук, 1979, т. 34, ном. 5, стр. 235-236.

[12] А. А. Шкаликов. Краевые задачи для обыкновенных дифференциальных уравнений с параметром в граничных условиях // Труды семинара им. И. Г. Петровского, 1983, вып. 9, стр. 190-229.

[13] А. А. Шкаликов. Эллиптические уравнения в гильбертовом пространстве и соответствующие спектральные задачи// Труды семинара им. И. Г. Петровского, 1983, вып. 14, стр. 140-224.

[14] Я. Д. Тамаркин. О некоторых задачах теории обыкновенных линейных дифференциальных уравнений и о разложении произвольных функиий в ряды// Петроград, 1917.

[15] А. А. Владимиров. О сходимости последовательностей обыкновенных дифференциальных операторов//Математические заметки, 2004, т. 75, вып. 6, с. 941-943. 